\title{
COVID-19 and biomarkers of thrombosis: focus on von Willebrand factor and extracellular vesicles
}

\author{
Richard C. Becker ${ }^{1}$ (D) Travis Sexton ${ }^{2} \cdot$ Susan Smyth ${ }^{3} \cdot$ for the International COVID-19 Thrombosis Biomarkers \\ Colloquium (ICODE) Investigators
}

Accepted: 15 July 2021 / Published online: 4 August 2021

(c) The Author(s), under exclusive licence to Springer Science+Business Media, LLC, part of Springer Nature 2021

\begin{abstract}
COVID-19, caused by the SARS-CoV-2 virus, is responsible for a pandemic of unparalleled portion over the past century. While the acute phase of infection causes significant morbidity and mortality, post-acute sequelae that can affect essentially any organ system is rapidly taking on an equally large part of the overall impact on human health, quality of life, attempts to return to normalcy and the global economy. Herein, we summarize the potential role of von Willebrand Factor and extracellular vesicles toward understanding the pathophysiology, clinical presentation, duration of illness, diagnostic approach and management of COVID-19 and its sequelae.
\end{abstract}

Keywords Thrombosis $\cdot$ Biomarkers $\cdot$ COVID-19 von Willebrand factor

\section{Highlights}

- COVID-19 has both acute and long-term clinical sequelae. Post-acute sequelae of SARS-CoV-2 infection or PASC is responsible for poor quality of life, impaired productivity, high health care costs and a serious economic impact. An ability to understand the pathophysiology, management and predictors of outcomes is of paramount importance.

\section{Introduction}

Circulating biomarkers may be useful in the diagnosis and management of patients with COVID-19 (reviewed in Grobler) [1]. Levels of vascular and thrombosis-related

Richard C. Becker

richard.becker@uc.edu

1 Heart, Lung and Vascular Institute, University of Cincinnati College of Medicine, 231 Albert Sabin Way, Cincinnati, OH 45267, USA

2 The Gill Heart and Vascular Institute, University of Kentucky, Lexington, KY, USA

3 University of Arkansas for Medical Sciences, Little Rock, AK, USA biomarkers, including those reflecting thrombin generation, fibrin formation, fibrin degradation, platelet activation and extracellular trap formation for example may offer insight for the acuity and stage of disease (reviewed in Becker) [2]. The potential role of biomarkers to gauge targeted therapies and to direct optimal management will require thorough investigation. The following review highlights the potential role of von Willebrand Factor, extracellular vesicles and plateletleukocyte aggregates in the pathophysiology of COVID-19 and potentially its management.

\section{von Willebrand factor}

\section{General constructs}

The dynamic nature of von Willebrand Factor (VWF), based on its sites of synthesis and storage in megakaryocytes, vascular endothelial cells (ECs) and platelets, reflects several concomitant events, including EC activation and injury, megakaryocyte mobilization, and platelet activation. The greater the stimulus for virus-induced cytotoxicity, proinflammatory cytokine production, cell-free nucleic acid-associated cellular injury and impaired VWF clearance mechanisms, the higher the level measured in peripheral blood (reviewed in Becker) [3]. What role might VWF play in portending clinical events, particularly thrombosis (arterial, 
venous, microvascular)? Should it be measured routinely? Might it represent target for treatment with a likelihood of improving clinical outcome?

\section{What is von Willebrand factor?}

Von Willebrand Factor (VWF) is a glycoprotein ranging in size from 600,000 to either 20 million Daltons that participates actively in platelet adhesion to injured, disrupted or activated ECs and high-shear stress associated platelet aggregation [4]. It also actively participates in both immune and inflammatory processes (described in subsequent sections).

\section{What is its origin?}

Megakaryocytes and ECs synthesize VWF. There are several distinct pathways of secretion. The first represents a constitutive pathway linked directly to synthesis. The second is a regulated pathway involving storage of mature molecules for release following stimulation by one or more mechanical, biochemical, cellular or protein mediators [5]. Weibel-Palade bodies (WPB), containing VWF are translocated to the cell surface of platelets and ECs following activation [6]. This property could be particularly important in the setting of COVID-19 [7].

Most VWF circulating in plasma originates within endothelial cell WPBs. The VWF multimer size and the length reflects the size of WPBs, typically ranging from 0.5 to $5.0 \mathrm{~mm}$, that is governed by the structural status of the Golgi apparatus where the organelles form.

\section{Structural and functional properties}

Protein sequencing of VWF has identified a 22-ammino acid hydrophobic signal peptide and a large pro-peptide that is identical to VWF antigen found within plasma. There are four homologous sequences or subunits of VWF. D repeats are found within each subunit and A repeats are in the central portion of each subunit (reviewed in Wagner) [8]. The binding sites for several ligands are localized to specific repeats of the VWF polypeptide. The crystal structures of VWFA1 and A3 repeats are known as are the three-dimensional structures of specific domains that consist of a series of short, alternating, $\alpha$-helical and $\beta$-pleated sheets that form a globular domain (reviewed in Ruggeri) [4].

The pre-pro-VWF molecule consists of a 22 -amino acid signal peptide, a 741 amino acid pro-peptide, and a 2050 amino acid mature subunit. The pro-vWF monomer is composed of four distinct domains (A-D). C-terminal and $\mathrm{N}$-terminal intermolecular disulphide bonds form VWF multimers. The largest multimers can exceed $2 \times 10^{4} \mathrm{kDa}$ and have the greatest overall functional activity. In the event of an ongoing stimulus for synthesis or release, the local concentrations of UL-VWF remain high. This is important to keep in mind when considering VWF targeted treatment in COVID-19.

\section{Regulatory mechanisms}

Fluid shear stress dynamically regulates VWF by promoting aggregation of multiple VWF units, while at the same time reducing multimer size through force-dependent cleavage by ADAMTS-13 [9]. Shear stress above 60 dyne $/ \mathrm{cm}^{2}$ is typically necessary to initiate VWF structural changes [10]. The unfolding and stretching of VWF increases in high shear stress environments that cause margination of platelets and platelet micro-particles toward the vessel wall $[3,11]$. VWF, like other plasma proteins has a relatively short circulating half-life, ranging from 6 to $24 \mathrm{~h}$ for multimers of smallto-moderate size or molecular weight [12]. The available evidence suggests that most VWF is cleared by an active regulatory mechanism involving the liver, spleen and ECs [13] with the former being determined by its co-localization with macrophages [14].

\section{What role might VWF play in COVID-19 phenotypes?}

\section{Pulmonary microvascular thrombosis}

The proclivity for thrombosis in patients with COVID-19 presents a broad array of pathophysiologic contributions for VWF; however, we believe that pulmonary microvascular thrombosis is likely the site of greatest impact [3]. Randomized clinical trials targeting VWF with pulmonary and respiratory endpoints such as oxygen support free days will be required as a "proof of concept" in early phase investigations.

\section{VWF and inflammation}

Vascular EC express procoagulant proteins, including factor VIII and VWF under inflammatory conditions (reviewed in Mazzeffi) [15]. The degree of inflammatory stress correlates with the concentrations of interleukin (IL)- 8 and tumor necrosis factor (TNF)- $\alpha$. Both cytokines trigger the release of UL-VWF multimers from EC in a concentrationdependent manner [16]. Vascular ECs under stress exhibit upregulation of adhesion receptors for leukocytes and platelets. Endothelial cell WPB maturation, secretion of adhesive proteins, and exocytosis increases substantially in such conditions [17]. In turn, VWF kinetics are impacted, leading to increased surface concentrations and availability for platelet, leukocyte and leukocyte-derived extracellular trap 
(ET) (primarily of neutrophil [N] origin or NETs) binding to injured or altered ECs.

\section{VWF anchors NETs to the vessel wall and to inflamed tissues}

NETs "trap" and kill bacteria [18], but they can also injure host tissues. VWF is believed to be a "linker molecule" for the binding of NETs to areas of vascular and tissue injury. The interaction between DNA and VWF takes place at the A1 domain of VWF [19]. The molecular mechanisms governing DNA-VWF interactions have been summarized by Sadoval-Perez et al. [20]. They have shown that doublestranded DNA binds to a specific helix of the VWF A1 domain via three arginine moieties. Shear stress and electrostatic guidance are the primary contributors.

\section{VWF and angiogenesis}

VWF is directly involved with maintaining normal vascular integrity. UL-VWF are the most important regulators of angiogenesis. Pathological angiogenesis occurs within the lungs of patients with severe COVID-19 (Reviewed in Becker) [3].

\section{VWF and hypoxia}

VWF expression is upregulated in heart and lung endothelial cells following hypoxia [21] and associates with the presence of thrombosis. Briefly, characterization of promotor and regulatory regions of the VWF gene are known to differ in EC located within the lung, heart, brain, and kidney vasculature. Upregulation in the lungs under hypoxic conditions occurs in both the micro-and macro-vasculature. The same is observed in EC of cardiac origin. In the absence of hypoxia VWF gene expression is confined to the brain and lung EC. VWF activation in lung EC also occurs in the presence of hypoxia [22]. The presence of megakaryocytes of bone marrow and spleen origin that reside in the pulmonary vasculature is a source of proplatelet release and platelet shedding in respond to local conditions including inflammation and hypoxia. The concomitant upregulation of VWF gene expression and activation collectively leads to a highly prothrombotic environment [23].

\section{VWF in COVID-19}

\section{Pathology and biomarker status: endothelial cell activation and injury}

Microvascular thrombosis is a hallmark of severe COVID19. Hottz et al. [24] found that platelet activation and platelet-monocyte heterotopic aggregates occurred more readily in patients with COVID-19 complicated by ARDS compared to those with lower acuity infection. They also reported that plasma procured from severe COVID-19 patients increased activation of control platelets ex-vivo. Last, platelet-monocyte interaction was strongly associated with monocyte tissue factor expression in both patients and healthy volunteers. The effect was neutralized by blocking $\mathrm{P}$-selection or the platelet $\alpha \mathrm{II} / \beta 3$ receptor.

Zaid et al. [25] reported a heightened platelet activation response to low concentrations of thrombin $(0.05 \mathrm{U} / \mathrm{ml})$ in patients with COVID-19 compared to patients with ARDS from non-COVID-19 related illness. Because thrombin mediated platelet activation (reviewed in Lundblad) [26] involves the GPIb $\alpha$ binding site with or without engagement with protease activated receptors (PARs), an association between thrombin and VWF functional dynamics is relevant in COVID-19 [27].

The experience with VWF and ADAMTS13 in COVID19 continues to grow, suggesting their potential role in identifying patients at risk for poor clinical outcomes following hospitalization (Table 1). Fraser et al. [28] characterized several markers of vascular EC and glycocalyx activation and injury in patients requiring admission to an Intensive Care Unit (ICU). Compared to healthy age and sex-matched controls, patients with COVID-19 had higher VWF, chondroitin sulfate, and syndecan levels. The level of each EC and glycocalyx biomarker was higher when compared to patients without COVID-19 in the ICU. Employing markers of thrombosis and EC injury, the investigators developed and trained a random forester identifier (machine-based learning) and identified predictors of mortality.

A single-center study of 68 hospitalized COVID-19 patients identified elevated VWF activity and antigen levels, particularly among those requiring ICU-level care [29], compared to non-hospitalized controls. VWF antigen $(\mathrm{r}=0.38$; $\mathrm{P}=0.001)$ and soluble $\mathrm{P}$-selection $(\mathrm{r}=0.38 ; \mathrm{P}=0.007)$, a marker of EC injury, correlated with in-hospital mortality.

Ladikou et al. reported biomarker profiles among 24 consecutive patients with COVID-19 admitted to the ICU [30]. The overall rate of venous thromboembolism and mortality was $25 \%$ and $16.7 \%$ respectively. Factor VIII and VWF levels were elevated at $279(251-363) \mu / \mathrm{dl}$ and $350(302-433)$ percent (median, IQR), respectively, and correlated with clinical events. VWF levels were higher in patients who did not survive their hospitalization (median 477\%) compared to those who survived $(335 \%)(\mathrm{P}=0.015)$.

Rauch et al. sampled 243 adults with COVID-19 at the time of hospital admission [31]. Increased VWF:Ag and decreased Factor VIII/VWF-Ag ratio were associated with increasing oxygen requirements in both a univariate and a multivariable analysis adjusted for age, sex, body mass index, hypertension and diabetes. In a series of 88 
Table 1 von Willebrand factor indices and clinical outcomes in observational studies

\begin{tabular}{|c|c|c|c|c|c|c|c|c|c|}
\hline \multirow[t]{2}{*}{ Investigator } & \multirow[t]{2}{*}{$\mathrm{N}$} & \multirow[t]{2}{*}{ Biomarker } & \multirow{2}{*}{ Primary origin } & \multirow{2}{*}{$\begin{array}{l}\text { Stimulus for } \\
\text { release }\end{array}$} & \multirow{2}{*}{$\begin{array}{l}\text { COVID-19 popu- } \\
\text { lation }\end{array}$} & \multicolumn{3}{|c|}{ Endpoints } & \multirow{2}{*}{$\begin{array}{l}\text { Strength of } \\
\text { Evidence }\end{array}$} \\
\hline & & & & & & ICU & Morbidity & Death & \\
\hline Fraser [27] & 10 & $\mathrm{VWF}_{\mathrm{Ag}} \uparrow$ & $\mathrm{EC}$ & Injury/activation & $\begin{array}{l}\text { Pneumonia } \\
\text { Hypoxia }\end{array}$ & $\checkmark$ & & & + \\
\hline Goshua [28] & 68 & $\mathrm{VWF}_{\mathrm{Ag}} \uparrow$ & $\mathrm{EC}$ & Injury/activation & $\begin{array}{l}\text { ICU } \\
\text { Non-ICU }\end{array}$ & & & $\checkmark$ & + \\
\hline Ladikou [29] & 24 & $\mathrm{VWF}_{\mathrm{Ag}} \uparrow$ & $\mathrm{EC}$ & Injury/activation & $\begin{array}{l}\text { ICU } \\
\text { High acuity ward }\end{array}$ & & VTE & $\checkmark$ & + \\
\hline Rauch [30] & 293 & $\mathrm{VWF}_{\mathrm{Ag}} \uparrow$ & $\mathrm{EC}$ & Injury/activation & Hospitalized & & $\begin{array}{l}\text { VTE } \\
\mathrm{O}_{2} \text { requirement }\end{array}$ & $\checkmark$ & ++ \\
\hline Bazzan [31] & 88 & $\begin{array}{l}\mathrm{VWF}_{\mathrm{Ag}} \uparrow \\
\mathrm{ADAMTS} 13 \downarrow\end{array}$ & $\begin{array}{l}\mathrm{EC} \\
\mathrm{EC}\end{array}$ & Injury/activation & Hospitalized & & & $\checkmark$ & ++ \\
\hline Masi [32] & 28 & $\begin{array}{l}\mathrm{VWF}_{\mathrm{Ag}} \mathrm{VWF} \\
\text { activity }\end{array}$ & $\mathrm{EC}$ & Injury/activation & ARDS & ND & ND & $\begin{array}{l}\text { ND } \\
\text { ND }\end{array}$ & $\begin{array}{l}- \\
-\end{array}$ \\
\hline Mancini [33] & 50 & $\begin{array}{l}\mathrm{VWF}_{\mathrm{Ag} \uparrow} \\
\mathrm{VWF}_{\text {activity }} \uparrow \\
\text { VWF propeptide } \uparrow \\
\text { VWF multimers } \uparrow \\
\text { ADAMTS13 activ- } \\
\text { ity } \downarrow \\
\text { VWF/ADAMTS13 } \\
\text { ratio } \uparrow\end{array}$ & $\begin{array}{l}\mathrm{EC} \\
\mathrm{EC}\end{array}$ & Injury/activation & $\begin{array}{l}\text { Hospital admis- } \\
\text { sion }\end{array}$ & $\mathrm{ND}$ & ND & & $\begin{array}{l}+ \\
+ \\
+ \\
+ \\
+ \\
+\end{array}$ \\
\hline Pascreau [36] & 70 & $\begin{array}{l}\mathrm{VWF}_{\text {activity }} \uparrow \\
\text { Multimers } \downarrow \\
\text { (HMW) } \\
\text { ADAMTS13 } \downarrow\end{array}$ & $\mathrm{EC}$ & Injury/activation & Pneumonia & $\begin{array}{l}\checkmark \\
\checkmark \\
\checkmark \\
\checkmark \\
\checkmark \\
\checkmark\end{array}$ & & & + \\
\hline Vassiliou [37] & 38 & $\mathrm{VWF}_{\mathrm{Ag}} \uparrow$ & $\mathrm{EC}$ & Injury/activation & ICU & & $\begin{array}{l}\checkmark \\
\text { Trend }\end{array}$ & & $+/-$ \\
\hline De Cristo [38] & 11 & $\begin{array}{l}\mathrm{VWF}_{\mathrm{Ag}} \uparrow \\
\mathrm{VWFactivity} \uparrow\end{array}$ & $\mathrm{EC}$ & Injury/activation & Hospitalized & & $\begin{array}{l}\checkmark \\
\text { Acuity }\end{array}$ & & + \\
\hline Fernandez [39] & 49 & $\begin{array}{l}\mathrm{VWF}_{\mathrm{Ag}} \uparrow \\
\text { ADAMTS13 activ- } \\
\text { ity } \downarrow\end{array}$ & $\mathrm{EC}$ & Injury/activation & Pneumonia & & $\begin{array}{l}\checkmark \\
\text { Sepsis }\end{array}$ & & + \\
\hline Thomas [40] & 143 & $\mathrm{VWF}_{\mathrm{Ag}} \uparrow$ & $\mathrm{EC}$ & Injury/activation & $\begin{array}{l}\text { Mild-to-severe } \\
\text { illness }\end{array}$ & & $\begin{array}{l}\checkmark \\
\text { Acuity }\end{array}$ & $\checkmark$ & ++ \\
\hline
\end{tabular}

+ low, ++ moderate,+++ high

$I C U$ Intensive care unit, $V W F$ Von Willebrand factor, $N D$ no difference, ADAMTS13 A disintegrin and metalloproteinase with a thrombospondin-like motif member 13, EC endothelial cell, $A R D S$ acute respiratory distress syndrome

consecutive cases of COVID-19. Bazzan et al. identified elevated levels of VWF: Ag and reduced levels of ADAMTS-13 compared to healthy controls. Patients who died had higher levels of VWF: Ag and lower levels of ADAMTS-13 than those who survived. In addition, an ADAMTS13 plasma level $<30 \%$ was associated with increased in-hospital mortality [32].

Masi et al. [33] described coagulation-related profiles in 28 consecutive patients with ARDS-17 patients had COVID19. Factor VIII: $\mathrm{C}$ was elevated in each patient with values 3-4-fold above the normal range, however, the values did not differ by infectious causes of ARDS.

Mancini and colleagues measured VWF antigen (VWF: Ag), VWF ristocetin-cofactor (VWF: RCo), VWF multimers, VWF propeptide (VWFpp) and ADAMTS13 activity in 50 patients stratified according to their intensity of care: low (requiring high-flow nasal cannula oxygenation, $\mathrm{n}=14$ ), intermediate (requiring continuous positive airway pressure devices, $\mathrm{n}=17$ ), high (requiring mechanical ventilation, $\mathrm{n}=19$ ). Median VWF: Ag, VWF: RCo and VWFpp levels were markedly elevated in COVID-19 patients and increased with intensity of care. By contrast, the high-tolow molecular weight VWF multimer ratios progressively decreased with increasing intensity of care [34].

Doevelaar and colleagues [35] measured VWF antigen, VWF multimers and ADAMTS13 in 75 patients with COVID-19. There was a marked increase in VWF antigen levels in patients compared with healthy controls, a higher 
ratio of VWF antigen to ADAMTS13, and loss of high molecular weight multimers with increasing severity of illness. A relative reduction in ADAMTS13 activity for the level of VWF antigen suggests a processing abnormality that could contribute to altered VWF multimer profiles and a heightened state of thrombosis potential. Plasma levels of ADAMTS13 inhibitors like IL-6, thrombospondin and platelet factor 4 are often elevated in patients with severe COVID-19 [36]. Other investigators and groups have drawn similar conclusions [37, 38-41].

Henry et al. [42] identified a lower ADAMTS13 activity/VWF:Ag ratio in patients with COVID-19 and acute kidney injury and those who developed infection compared to patient with neither. In a prospective study of 50 patients with COVID-19 requiring hospital admission [34], higher D-dimer, fibrinogen and VWF:Ag levels and lower ADAMTS13 activity were observed compared to patients not requiring hospitalization. Patients requiring ICU-level care had the lowest ADAMTS13 activity.

\section{VWF and venous thrombosis}

The vasculopathy described in COVID-19 produces an environment favoring UL-VWF-platelet thrombosis either in situ or in the form of emboli that lodge within the microvasculature of vital organs-most often the lungs [43]. Morici et al. [44] reported six patients with COVID-19-one with subclavian and axillary vein thrombosis and two with bleeding events. Factor VIII, VWF:Ag, VWF:RCo and VWF:CB were elevated in each patient. Five of six patients had ADAMTS13 levels below 45\% (lower limit of normal) and three had levels of $\sim 30 \%$. Plasma levels of antibodies against ADAMTS 13 were not detectable in any of the patients. Factor VIII and VWF levels were elevated at $279(251-363) \mu$ / $\mathrm{dl}$ and 350 (302-433) percent (median, IQR), respectively, and correlated with VTE in the study by Ladikou et al. [30].

\section{Extracellular vesicles}

Extracellular vesicles (EV) are lipid bilayer-encapsulated particles that are released by cells and transfer proteins, lipids, metabolites, nucleic acids, and organelles to other cells, often in tissues at distant sites of the body. The release of VWF from platelets and ECs directly correlates with the release of $E V$ from the same origins. Various functions have been attributed to EV, including promotion of cell-cell signaling, elimination cellular waste or molecular recycling, and mediating host-pathogen interactions. EV are heterogenous in nature, ranging in size from $20 \mathrm{~nm}(\mathrm{~nm})$ to $1 \mu \mathrm{m}$, and can be distinguished based on size with exosomes being on the low end and micro vesicles (previously referred to as microparticles) on the high end of the size range. EV are found in many biologic fluids such as plasma, urine, bile, saliva, bronchoalveolar lavage fluid, ascites, and lymph. In circulation, they can be released in response to pro-thrombotic conditions, inflammation, shear stress, and complement activation.

Platelets, megakaryocytes, leukocytes, endothelial cells and red blood cells all release EV, and may be influenced by EV-mediated effects. Platelet-derived EVs can originate from the plasma membrane of resting platelets or from alpha granules following platelet activation. Endothelialand monocyte-derived EV express selectins, tissue factor, VWF, other coagulation factors, and negatively charged phosphatidyl serine that can promote thrombosis (reviewed in $\mathrm{Fu}$ ) [45]. Circulating EV may also regulate inflammation and vascular permeability ([46]). Weibel-Palade derived small EV have also been found in circulation [47] and have a distinct molecular signature (CD36+/CD9-/CD81-) that distinguishes them from other small EV (CD36+/CD9+/ CD81+).

\section{Extracellular Vesicles and COVID-19}

Severe acute respiratory syndrome, such as can occur with SARS-CoV-2 infection, has been associated with the development of autoantibodies [48] that may stimulate the classical pathway of complement activation to trigger the release of EV [49, 50]. In the context of COVID-19, it has been speculated that EV could regulate angiotensin converting enzyme 2, which is a key protein that is recognized by the SARS-CoV2 spike protein and mediates cell entry of the virus [51, 52]. By regulating proteins important for cellular entry of the SARS-CoV2 virus, EV might also regulate viral pathogenesis and disease severity (Fig. 1).

Infection with SARS-CoV-2 can lead to both endothelial dysfunction and dysregulated immune responses, conditions that are known to be associated with the generation of EV. In an analysis of plasma from 53 patients hospitalized with COVID-19 [53], Krishnamachary et al. characterized large $(100-300 \mathrm{~nm})$ and small $(30-100 \mathrm{~nm}) \mathrm{EV}$, obtained by differential centrifugation of plasma at $20,000 \times g$ and $100,000 \times g$ respectively. The total number of small and large EV increased with disease severity, as did the mean particle size. Analysis of protein cargo in the large EV demonstrated distinct differences based on disease severity, with specific changes observed in the IL6 family and TNF $\alpha$ and TNF-receptor superfamily proteins. Prothrombotic factors within the large EV were also higher. In another study of 19 patients with COVID-19 pneumonia that were followed for 14 days for the development of severe respiratory failure [54], procoagulant phospholipid levels were higher in patients than healthy controls. No difference was observed in procoagulant phospholipid activity between patients with severe $\left(\mathrm{PO}_{2} / \mathrm{FiO}_{2}<200\right)$ and non-severe pneumonia, although 




Fig. 1 The migration of inflammatory cells, specifically monocytes is responsible for a number of proinflammatory and prothrombotic steps, including tissue factor expression, cytokine production and along with neutrophils and their interaction with platelets, NETosis,

higher procoagulant phospholipid levels on admission were associated with to severe respiratory failure, suggesting that they may be a marker for disease course.

In support of a role of $\mathrm{EV}$ in promoting thrombosis in COVID-19, Rosell et al. [55] demonstrated that circulating EV expressing tissue factor (TF) correlated with disease severity. They studied 100 patients with COVID-19 in comparison to 28 healthy controls. Levels of EV-associated TF activity were higher in COVID-19 patients than controls, higher in patients that required $>5 \mathrm{~L} \mathrm{O}_{2} / \mathrm{min}$, and higher in non-survivors. Anticoagulation use did not affect levels of EV-associated TF activity nor did treatment with corticosteroids. EV-associated TF activity strongly correlated with D-dimer levels, a marker for thrombosis in this patient population. EV levels also correlated with prothrombin time, fibrinogen levels, plasmin-antiplasmin complexes, von Willebrand factor, and ADAMTS13. Guervilly et al. [56], also demonstrated that EV-associated TF activity increased with disease severity and correlated with leukocytes, D-dimer, and inflammatory parameters. This study protein expression, ROS production and recruitment of ULVWF to the endothelial cell surface. NET neutrophil extracellular traps, $V W F$ von Willebrand Factor, ROS reactive oxygen species

further compared severe COVID patients to non-COVID septic shock patients and found that patients with COVID19 had higher coagulopathy proles with significantly higher EV-TF activity.

Flow cytometry has been used to identify platelet-derived $\mathrm{EV}$ in plasma from patients with COVID-19 [57] using a cationic dye to bind the membrane, phalloidin to identify intact vesicles, and antibodies to platelet-specific CD41a (integrin $\alpha I I b \beta 3$ ) or platelet and endothelial expressed CD31 (PECAM). Platelet-derived EV were higher in 2 cohorts of patients hospitalized with symptoms in April/May of 2020 $(n=23)$ or October/November of $2020(n=46)$ than in COVID-negative patients hospitalized during the same two time periods $(n=62)$ or healthy controls $(n=10)$. The results are in keeping with the findings of Taus et al. ([58]) who reported that platelet-derived vesicles, identified by expression of CD41 (integrin $\alpha \mathrm{IIb}$ ) and ranging in size from 100 to $1000 \mathrm{~nm}$, were elevated in patients with patients with COVID-19 $(n=17)$ versus healthy controls $(n=22)$. Finally, Zaid et al. [25] noted increased numbers of platelet-derived 
EV, identified by CD41, in both patients with severe $(n=44)$ and non-severe $(\mathrm{n}=71)$ COVID-19 infection in comparison to healthy controls $(n=18)$. The total levels of plateletderived EV were highest in the patient cohort with nonsevere disease, and levels of phosphatidylserine-expressing platelet EV was only higher in patients with non-severe COVID-19.

Given the accumulated observed presence of EVs during COVID-19 and the long-known pro-inflammatory and prothrombotic nature of EVs, it is very likely that MVs play an important role in the progression and severity of the disease and therefore could be an important reporter or target for treatment in the future.

\section{Platelet-leukocyte interactions}

Under certain conditions, platelets are known to interact with a variety of cell types and tissues that they are exposed to circulating blood. Thus, the role that platelets play extends well beyond their well-known role in hemostasis. Among the cell types that platelets will interact with are leukocytes, primarily monocytes and neutrophils. These interactions are pro-inflammatory and platelet-leukocyte interactions have served as an indicator of in vivo platelet activation as well as a strong predictor of major adverse cardiovascular events (MACE). Circulating platelet-leukocyte aggregates and extracellular vesicles increase in animal models of lung injury [59]. Furthermore, platelet-leukocyte interactions are known to be increased during lung infections and antiplatelet therapy has been shown to drive down platelet-leukocyte interactions as well inflammatory biomarkers [60].

\section{Platelet-leukocyte interactions in COVID-19}

Platelet hyperactivation in patients with COVID-19 has been reported by several groups [24, 25, 58, 61]. Platelet-leukocyte interactions contribute to the pathophysiology of some viral infections and may play a similar role in SARS-CoV-2 infection. Expression of platelet P-selectin, an $\alpha$-granule protein that undergoes translocation to the plasma membrane with platelet activation where is mediates platelet-leukocyte interactions, was higher patients with COVD-19. Platelet-monocyte and platelet-neutrophil aggregates as detected by flow cytometry were significantly higher in patients than healthy controls. In addition, an analysis of blood smears from COVID-19 patients revealed platelet engulfment by lymphocytes [58]. Leopold et al. [61] also found higher levels of platelet-neutrophil aggregates in patients hospitalized with COVID-19 $(n=35)$. In their study of COVID-19 patients within $72 \mathrm{~h}$ of ICU admission $(\mathrm{n}=35)$, Hottz et al. [24] found higher levels of platelet-monocyte aggregates in ICU patients than asymptomatic/mildly symptomatic patients or healthy controls. In addition, TF expression by monocytes with attached platelets was significantly higher than in monocytes alone. Monocyte TF expression correlated with D-dimer levels, suggesting a potential role for platelet-stimulation of monocyte TF expression in thrombosis associated with COVID-19. Indeed, incubation of monocytes from healthy controls with platelets from patients with severe COVID-19 resulted in enhanced expression of monocyte $\mathrm{TF}$ in a manner that required platelet $\mathrm{P}$-selectin and integrin $\alpha \operatorname{IIb} \beta 3$. In addition to promoting TF exposure and thrombin generation, platelet-leukocyte interactions can also contribute to neutrophil extracellular traps, cytokine release, generation of reactive oxygen species, and formation of novel transcellular metabolites of arachidonic acid [62] — each could promote inflammation and thrombosis in the setting of COVID-19.

\section{Diagnostic assays}

\section{Peripheral blood}

Peripheral blood diagnostic assays for VWF include initial screening, first level tests and second level tests. First level tests are widely available for the evaluation of patients with COVID-19. They include the measurement of plasma FVIII coagulant activity (FVIII:C), VWF antigen (VWF:Ag) and platelet-dependent VWF activity, the latter being commonly measured as ristocetin cofactor (VWF:RCo) that evaluates the ability of plasma VWF to agglutinate platelets in the presence of ristocetin. The ristocetin-triggered GPIb $\alpha$ binding (VWF:GPIbR) assay uses ristocetin and a GPIb $\alpha$ fragment captured by a monoclonal antibody coated onto an ELISA plate or latex or magnetic particles (for enhanced automated assays). The routine measurement of VWF antigen, activity or ADAMTS13 activity is not recommended at this time. The same is currently true for measuring EV and platelet- leukocyte aggregates.

\section{Targeted treatment options}

\section{Scientific premise}

ADAMTS 13 cleaves VWF at its A2 domain. Accordingly, a therapy designed to inhibit the binding or catalytic activity of ADAMTS13 could prevent cleavage and the resulting down-stream effects. There are several challenges and considerations with such a strategy, including (1)VWF, under normal circumstances, is metabolized with a circulating half-life of 12-20 h, and (2) preventing either the binding or catalytic activity of ADAMTS13 could cause an excess of UL-VWF multimers and provoke the equivalent of thrombotic thrombocytopenic purpura (TTP) — a highly prothrombotic disorder [63]. Targeted therapies have not yet been tested in COVID-19. 


\section{VWF nanobody}

\section{Caplacizumab}

Caplacizumab is a bivalent, humanized, single-variable domain immunoglobulin of nanobody that targets the A1 domain of VWF (reviewed in Knoebl) [64]. The drug inhibits the interaction between UL-VWF multimers and platelets. The drug is FDA-approved for the management of thrombotic thrombocytopenic purpura, including patients with refractory or recurrent disease [65].

Caplacizumab administration is subcutaneous after an initial intravenous loading dose. Cmax occurs within an hour [64] and binding to free VWF occurs rapidly and selectively. The caplacizumab-VWF complex circulates to the liver where it degrades within the reticuloendothelial system. Free (unbound) caplacizumab distributes to several organs where it is proteolytically degraded. The fraction of an administered dose recovered in the urine is small. The mean terminal half-life is $38.5 \pm 22.2 \mathrm{~h}$ in healthy volunteers and is dose-proportional. A single $10 \mathrm{mg}$ dose administered subcutaneously produced complete ristocetin-induced cofactor activity for $24 \mathrm{~h} \mathrm{[64].} \mathrm{It} \mathrm{is} \mathrm{currently} \mathrm{approved} \mathrm{for} \mathrm{the}$ treatment of thrombotic thrombocytopenic purpura.

\section{Aptamers}

Aptamers are single-stranded oligonucleotides that have theoretical advantages over other classes of therapeutic agents. They bind to their target with high affinity, in the low nanomolar to high picomolar range, like monoclonal antibodies.

\section{BT200}

BT200 is a pegylated RNA aptamer that inhibits binding of VWF to platelet GPIb [66]. Its target is the A1 domain and the circulating half-life exceeds $100 \mathrm{~h}$ following subcutaneous injection [67].

\section{TAGX-0004}

TAGX-0004 is a DNA aptamer developed to target the VWF A1 domain [68]. It contains DS—an artificial nucleic acid that has no complimenting base in nature, permitting unique 3D structures and enhancing its target binding affinity.

\section{DTRI 031}

DTRI-031 is a 36-nucleotide RNA aptamer that targets the A1 binding domain of VWF. It consists of a single stranded RNA. A matched reversal agent, DTRI-025, a sequence specific oligonucleotide that neutralizes the pharmacologic effect of DTRI-031 ( $<5 \mathrm{~min})$ is undergoing IND enabling studies. Preclinical testing with DTRI-031 has been undertaken $[69,70]$.

\section{Cationic polymers}

An ability to target thrombus-associated NETs bound to injured or disrupted ECs by VWF could represent a novel, yet viable option for the treatment of COVID-19-related thrombosis, particularly within the microvasculature. Preliminary evidence from Jain et al. [71] suggests that an approach to therapeutics based on binding of cationic polymers is feasible.

\section{Recombinant ADAMTS13}

The observed increase in VWF, coupled with decreased ADAMTS13 activity in COVID-19 lends itself to potential treatment options designed to restore VWF homeostasis. Turecek and colleagues incubated plasma samples obtained from 36 patients with severe COVID-19 with recombinant ADAMTS13 [72]. They observed a decrease in VWF activity and restoration of a normal pattern of multimer size.

\section{Conclusions and future directions}

The COVID-19 pandemic emphasizes the importance of understanding the pathobiology of disease, gauging the severity and natural history of disease with readily available and reproducible tools and making decisions about treatment and overall management according to the best available evidence. Circulating biomarkers of thrombosis, including VWF and extracellular vesicles have emerged and based on the information available may offer prognostic insights during the early phase of COVID-19. The question remaining is whether either represents a worthy target for therapy that will favorably influence clinical outcomes of patients with COVID-19.

\section{References}

1. Grobler C et al (2020) (2020) Covid-19: the rollercoaster of fibrin(ogen), D-dimer, Von Willebrand factor, P-selectin and their 
interactions with endothelial cells, platelets and erythrocytes. Int J Mol Sci 21(14):5168

2. Becker RC (2021) COVID-19 and its sequelae: a platform for optimal patient care, discovery and training. J Thromb Thrombolysis 51:587-594

3. Becker RC (2020) COVID-19 update: Covid-19-associated coagulopathy. J Thromb Thrombolysis 50(1):54-67

4. Ruggeri ZM (2000) Role of von Willebrand factor in platelet thrombus formation. Ann Med 32(Suppl 1):2-9

5. Huisman B et al (2017) Modeling the cleavage of von Willebrand factor by ADAMTS13 protease in shear flow. Med Eng Phys 48:14-22

6. Sadler JE (1998) Biochemistry and genetics of von Willebrand factor. Annu Rev Biochem 67:395-424

7. Becker RC (2020) COVID-19-associated vasculitis and vasculopathy. J Thromb Thrombolysis 50:499-511

8. Wagner DD, Bonfanti R (1991) von Willebrand factor and the endothelium. Mayo Clin Proc 66(6):621-627

9. Lynch CJ, Lane DA, Luken BM (2014) Control of VWF A2 domain stability and ADAMTS13 access to the scissile bond of full-length VWF. Blood 123(16):2585-2592

10. Bharati KP, Prashanth UR (2011) Von Willebrand disease: an overview. Indian J Pharm Sci 73(1):7-16

11. Rack K, Huck V (2017) Margination and stretching of von Willebrand factor in the blood stream enable adhesion. Sci Rep $7(1): 14278$

12. Sztukowska M et al (2008) Von Willebrand factor propeptide makes it easy to identify the shorter Von Willebrand factor survival in patients with type 1 and type Vicenza von Willebrand disease. Br J Haematol 143(1):107-114

13. van Schooten CJ et al (2008) Macrophages contribute to the cellular uptake of von Willebrand factor and factor VIII in vivo. Blood 112(5):1704-1712

14. Casari C et al (2013) Accelerated uptake of VWF/platelet complexes in macrophages contributes to VWD type 2B-associated thrombocytopenia. Blood 122(16):2893-2902

15. Mazzeffi MA, Chow JH, Tanaka K (2020) COVID-19 associated hypercoagulability: manifestations, mechanisms, and management. Shock 55:465-471

16. Bernardo A et al (2004) Effects of inflammatory cytokines on the release and cleavage of the endothelial cell-derived ultralarge von Willebrand factor multimers under flow. Blood 104(1):100-106

17. Holthenrich A et al (2019) Proximity proteomics of endothelial Weibel-Palade bodies identifies novel regulator of von-Willebrand factor secretion. Blood 134:979-982

18. Brinkmann V (2018) Neutrophil extracellular traps in the second decade. J Innate Immun 10(5-6):414-421

19. Grassle $\mathrm{S}$ et al (2014) von Willebrand factor directly interacts with DNA from neutrophil extracellular traps. Arterioscler Thromb Vasc Biol 34(7):1382-1389

20. Sandoval-Pérez A et al (2020) DNA binds to a specific site of the adhesive blood-protein von Willebrand factor guided by electrostatic interactions. Nucleic Acids Res 48(13):7333-7344

21. Mojiri A et al (2019) Endothelial cells of different organs exhibit heterogeneity in von Willebrand factor expression in response to hypoxia. Atherosclerosis 282:1-10

22. Mojiri A et al (2013) Hypoxia results in upregulation and de novo activation of von Willebrand factor expression in lung endothelial cells. Arterioscler Thromb Vasc Biol 33(6): 1329-1338

23. Lefrançais E, Looney MR (2019) Platelet biogenesis in the lung circulation. Physiology (Bethesda) 34(6):392-401

24. Hottz ED et al (2020) Platelet activation and platelet-monocyte aggregate formation trigger tissue factor expression in patients with severe COVID-19. Blood 136(11):1330-1341
25. Zaid Y et al (2020) Platelets can associate with SARSCov-2 RNA and are hyperactivated in COVID-19. Circ Res 127(11):1404-1418

26. Lundblad RL, White GC (2005) The interaction of thrombin with blood platelets. Platelets 16(7):373-385

27. Dörmann D, Clemetson KJ, Kehrel BE (2000) The GPIb thrombin-binding site is essential for thrombin-induced platelet procoagulant activity. Blood 96(7):2469-2478

28. Fraser DD et al (2020) Endothelial injury and glycocalyx degradation in critically ill coronavirus disease 2019 patients: implications for microvascular platelet aggregation. Crit Care Explor 2(9):e0194

29. Goshua G et al (2020) Endotheliopathy in COVID-19-associated coagulopathy: evidence from a single-centre, cross-sectional study. Lancet Haematol 7(8):e575-e582

30. Ladikou EE et al (2020) Von Willebrand factor (vWF): marker of endothelial damage and thrombotic risk in COVID-19? Clin Med (Lond) 20(5):e178-e182

31. Rauch A et al (2020) Coagulation biomarkers are independent predictors of increased oxygen requirements in COVID-19. J Thromb Haemost 18:2942-2953

32. Bazzan M et al (2020) Low ADAMTS 13 plasma levels are predictors of mortality in COVID-19 patients. Intern Emerg Med 15(5):861-863

33. Masi P et al (2020) Systemic inflammatory response syndrome is a major contributor to COVID-19-associated coagulopathy: insights from a prospective, single-center cohort study. Circulation 142(6):611-614

34. Mancini I et al (2021) The ADAMTS13-von Willebrand factor axis in COVID-19 patients. J Thromb Haemost 19(2):513-521

35. Doevelaar AAN et al (2021) von Willebrand factor multimer formation contributes to immunothrombosis in coronavirus disease 2019. Crit Care Med 49(5):e512-e520

36. Ward SE et al (2021) ADAMTS13 regulation of VWF multimer distribution in severe COVID-19. J Thromb Haemost. https://doi. org/10.1111/jth.15409

37. Pascreau T et al (2021) ADAMTS 13 deficiency is associated with abnormal distribution of von Willebrand factor multimers in patients with COVID-19. Thromb Res 204:138

38. Vassiliou AG et al (2021) ICU admission levels of endothelial biomarkers as predictors of mortality in critically Ill COVID-19 patients. Cells 10(1):18

39. De Cristofaro R et al (2021) Marked von Willebrand factor and factor VIII elevations in severe acute respiratory syndrome coronavirus-2-positive, but not severe acute respiratory syndrome coronavirus-2-negative, pneumonia: a case-control study. Blood Coagul Fibrinolysis. https://doi.org/10.1097/MBC.0000000000 000998

40. Fernández S et al (2021) Distinctive biomarker features in the endotheliopathy of COVID-19 and septic syndromes. Shock. https://doi.org/10.1097/SHK.0000000000001823

41. Thomas VV et al (2021) Plasma Von Willebrand factor levels predict survival in COVID-19 patients across the entire spectrum of disease severity. Indian J Hematol Blood Transf. https://doi. org/10.1007/s12288-021-01459-0

42. Henry BM et al (2020) ADAMTS13 activity to von Willebrand factor antigen ratio predicts acute kidney injury in patients with COVID-19: Evidence of SARS-CoV-2 induced secondary thrombotic microangiopathy. Int J Lab Hematol 43:129

43. Varatharajah N, Rajah S (2020) Microthrombotic complications of COVID-19 are likely due to embolism of circulating endothelial derived ultralarge von willebrand factor (eULVWF) decoratedplatelet strings. Fed Pract 37(6):258-259

44. Morici N et al (2020) Role of von Willebrand factor and ADAMTS-13 in the pathogenesis of thrombi in SARS-CoV-2 infection: time to rethink. Thromb Haemost 120(9):1339-1342 
45. Fu S et al (2020) Extracellular vesicles in cardiovascular diseases. Cell Death Discov 6:68

46. Chatterjee V et al (2020) Extracellular vesicles: new players in regulating vascular barrier function. Am J Physiol Heart Circ Physiol 319(6):H1181-h1196

47. Streetley J et al (2019) Stimulated release of intraluminal vesicles from Weibel-Palade bodies. Blood 133(25):2707-2717

48. Yang YH et al (2005) Autoantibodies against human epithelial cells and endothelial cells after severe acute respiratory syndrome (SARS)-associated coronavirus infection. J Med Virol 77(1):1-7

49. Inal J (2020) Complement-mediated extracellular vesicle release as a measure of endothelial dysfunction and prognostic marker for COVID-19 in peripheral blood - letter to the Editor. Clin Hemorheol Microcirc 75(4):383-386

50. Inal J (2020) COVID-19 comorbidities, associated procoagulant extracellular vesicles and venous thromboembolisms: a possible link with ethnicity? Br J Haematol 190(4):e218-e220

51. Marchandot B et al (2020) COVID-19 related coagulopathy: a distinct entity? J Clin Med 9(6):1651

52. Hassanpour $\mathrm{M}$ et al (2020) The role of extracellular vesicles in COVID-19 virus infection. Infect Genet Evol 85:104422

53. Krishnamachary B, et al (2020) The potential role of extracellular vesicles in COVID-19 associated endothelial injury and proinflammation. medRxiv

54. Assimakopoulos SF et al (2020) Evidence for increased circulating procoagulant phospholipids in patients with COVID-19 pneumonia and their prognostic role. Clin Chem Lab Med 59(2):e53-e55

55. Rosell A et al (2020) Patients with COVID-19 have elevated levels of circulating extracellular vesicle tissue factor activity that is associated with severity and mortality. Arterioscler Thromb Vasc Biol 41(2): 120315547

56. Guervilly C et al (2021) Dissemination of extreme levels of extracellular vesicles: tissue factor activity in patients with severe COVID-19. Blood Adv 5(3):628-634

57. Cappellano $\mathrm{G}$ et al (2021) Circulating platelet-derived extracellular vesicles are a hallmark of SARS-COV-2 infection. Cells 10(1):85

58. Taus F et al (2020) Platelets promote thromboinflammation in SARS-CoV-2 pneumonia. Arterioscler Thromb Vasc Biol 40(12):2975-2989

59. McVey MJ et al (2021) Platelet extracellular vesicles mediate transfusion-related acute lung injury by imbalancing the sphingolipid rheostat. Blood 137(5):690-701

60. Gross AK et al (2013) Clopidogrel treatment and the incidence and severity of community acquired pneumonia in a cohort study and meta-analysis of antiplatelet therapy in pneumonia and critical illness. J Thromb Thrombolysis 35(2):147-154

61. Léopold V et al (2021) Platelets are hyperactivated but show reduced glycoprotein VI reactivity in COVID-19 patients. Thromb Haemost. https://doi.org/10.1055/a-1347-5555

62. Becker RC, Sexton T, Smyth SS (2018) Translational implications of platelets as vascular first responders. Circ Res 122(3):506-522

63. Mehta R et al (2019) Acquired Von Willebrand syndrome (AVWS) in cardiovascular disease: a state of the art review for clinicians. J Thromb Thrombolysis 48(1):14-26

64. Knoebl P et al (2020) Efficacy and safety of open-label caplacizumab in patients with exacerbations of acquired thrombotic thrombocytopenic purpura in the HERCULES study. J Thromb Haemost 18(2):479-484

65. Völker LA et al (2020) ADAMTS13 and VWF activities guide individualized caplacizumab treatment in patients with aTTP. Blood Adv 4(13):3093-3101

66. Zhu S et al (2020) The development and characterization of a long acting anti-thrombotic von Willebrand factor (VWF) aptamer. J Thromb Haemost 18(5):1113-1123

67. Zhu S et al (2020) Potent and rapid reversal of the von Willebrand factor inhibitor aptamer BT200. J Thromb Haemost 18(7):1695-1704

68. Kazuya S et al (2019) Novel aptamer to von Willebrand factor A1 domain (TAGX-0004) shows total inhibition of thrombus formation superior to ARC1779 and comparable to caplacizumab. Haematologica 105(11):2631-2638

69. Nimjee SM et al (2019) Preclinical development of a vWF aptamer to limit thrombosis and engender arterial recanalization of occluded vessels. Mol Ther 27(7):1228-1241

70. Nimjee SM et al (2019) Preclinical development of a vWF Aptamer to limit thrombosis and engender arterial recanalization of occluded vessels. Mol Ther 27(7):1228-1241

71. Jain $S$ et al (2012) Nucleic acid scavengers inhibit thrombosis without increasing bleeding. Proc Natl Acad Sci U S A 109(32):12938-12943

72. Turecek PL et al (2021) Recombinant ADAMTS13 reduces abnormally up-regulated von Willebrand factor in plasma from patients with severe COVID-19. Thromb Res 201:100-112

Publisher's Note Springer Nature remains neutral with regard to jurisdictional claims in published maps and institutional affiliations. 